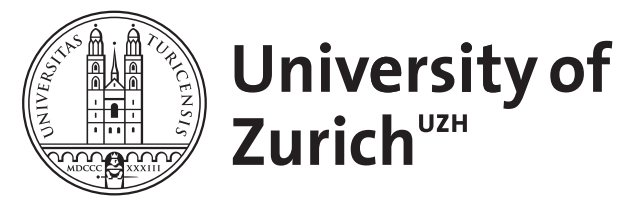

\title{
Hat die Philosophie noch eine Einheit?
}

Leist, Anton

\begin{abstract}
Today philosophy falls apart into a "theoretical" and a "practical" sub-discipline, a situation to the detriment of both. On the back of a sketch of three stages of Western philosophy a diagnosis is made as to the practical origin of the theoretical/practical split, which also already contains within it the clue for a therapy. Both parts of philosophy could be set on a convergent path by recognizing two practical conflicts underlying philosophical problems: the biographical conflict of accepting the world and the social conflict of accepting others. A non-reductivist study of these conflicts might mitigate the present alienation within philosophy
\end{abstract}

DOI: https://doi.org/10.1524/dzph.2012.0049

Posted at the Zurich Open Repository and Archive, University of Zurich ZORA URL: https://doi.org/10.5167/uzh-154599

Journal Article

Published Version

Originally published at:

Leist, Anton (2012). Hat die Philosophie noch eine Einheit? Deutsche Zeitschrift für Philosophie, 60(5):643-657.

DOI: https://doi.org/10.1524/dzph.2012.0049 


\title{
Hat die Philosophie noch eine Einheit?*
}

\author{
Von ANTON LEIST (Zürich)
}

\section{Die peinliche Frage}

Die meisten der Philosophie Unterrichtenden kennen den Reflex ihrer eigenen Hilflosigkeit, wenn sie der Erwartung von Anfängern in der Philosophie begegnen, dass die Philosophie ihr Leben bereichere. Die herrschende Doktrin bei den Unterrichtenden ist wohl mehrheitlich die, dass die Philosophie eine Art Wissenschaft sei, die Philosophen eine Art Wissenschaftler, und die Frage nach dem Bereichern deshalb in den privaten Raum gehört, wie eben auch bei einem Physikstudenten, der für sich selbst entdecken muss, ob die Suche nach Higgs-Teilchen zu seinem Glück beitragen wird oder nicht.

Ein solcher reflexhafter Vergleich von Philosophie und Physik ist, schon weil er nicht selten ist, nicht unmöglich. Man muss sich aber bewusst sein, dass er Folgen für das Verständnis der Philosophie hat. Er kann zur Folge haben, dass die Philosophie überwiegend zur Philosophiegeschichte wird, über die wissenschaftlich, eben historisch, gearbeitet wird. Wird die Philosophie hingegen dennoch mit dem eigenständigen, „systematischen“ Anspruch betrieben, scheint das den Bezug zu einem sinnvollen oder guten Leben aufzulösen. Weil die meisten Philosophen weder die erste noch die zweite Folge akzeptieren wollen, retten sie sich in der Auskunft gegenüber einem Studenten in der Regel mit einer unverfänglich schwachen Version des Lebensbezugs. Das Studium der Philosophie biete, so die übliche, banalst-denkbare Antwort, die Möglichkeit, „,komplexe Zusammenhänge“ im Denken beherrschen zu lernen.

Vielleicht ist das ein zu hartes Urteil. Ein Studienberater, von einer Anfängerin in der Philosophie um Motive und Nützlichkeiten bedrängt, wird heute bei anhaltender Nachfrage von Seiten der Studentin irgendwann die Trumpfkarte der Ethik ausspielen und auf die bedeutsame Rolle der Philosophen beim Lösen vieler sozialpolitisch relevanter Konflikte in der demokratischen Öffentlichkeit hinweisen. Diese Antwort ist nicht unberechtigt und stützt sich auf ein disziplinäres Binnenverständnis der gegenwärtigen Philosophie, die ihr klassisches Teilfach der Ethik auf zahlreiche Themen der ,angewandten Ethik“ erweitert hat. Befriedigend ist diese Antwort auf die Frage nach der Motivation, Philosophie zu studieren, aber ebenfalls nicht. Wenn die fragende Anfängerin ihrem zu wählenden Studium würdig ist, wird sie nämlich zum Zusammenhang der Ethik mit der restlichen Philosophie weiterfragen. Sie will ja nicht nur Ethik, sondern Philosophie insgesamt studieren. Außerdem habe

\footnotetext{
Abschiedsvorlesung an der Universität Zürich am 31. Mai 2012.
} 
sie beobachtet, dass auch Theologen und Juristen, neuerdings sogar Mediziner, Biologen und Ökonomen ethische Fachleute seien. Was sei dann das Philosophische an der Ethik?

Das Fehlen klarer Antworten bei diesen Motivationsfragen ist nicht nur ein Problem für die Anfänger und deren Berater, die noch nach einem Motiv suchen. Es ist auch ein Problem für die Philosophie, weil es ein Symptom zweier Bruchlinien ist, die die Philosophie als ein systematisches Arbeitsgebiet bedrohen und sie als Disziplin grundsätzlich in Frage stellen. Beide Bruchlinien sind solche zwischen ,dem Theoretischen und dem Praktischen“, und sie stellen in diesem Sinn die innere Einheit der Philosophie in Frage. Die von der Studentin erfragte Einheit ist einerseits die zwischen den in der alltäglichen Lebenseinstellung enthaltenen Motiven und der Philosophie, und andererseits die zwischen dem empirischen Wissen und dem praktischen Handeln generell.

Die in der Philosophie geläufige Terminologie fasst den ersten Zusammenhang als den zwischen dem „,sinnvollen“ oder „guten Leben“ und der Philosophie, den zweiten als den zwischen theoretischer und praktischer Philosophie. Auch ungeachtet historischer Kenntnisse der früheren, vorrangig in der Antike bestehenden Einheit scheint klar, dass die Philosophie in Schwierigkeiten ist, wenn sie zwischen diesen drei Teilen - gutes Leben, theoretische Reflexion, Ethik - keinen überzeugenden Zusammenhang bewahren kann. Wie real diese Schwierigkeiten sind, zeigt sich sowohl an der fortwährenden Pluralisierung der angewandten Ethik durch Nichtphilosophen als auch an der naturalistischen Transformation klassischer Teile der theoretischen Philosophie, gegenwärtig insbesondere derjenigen des Geistes und des Erkennens. Der Tag könnte nicht allzu fern sein, an denen Philosophen an Konferenzen zu diesen Themen, wie bereits in der angewandten Ethik, nur Experten unter anderen sind.

\section{Philosophische Reaktion}

Um die Schwierigkeiten, aber auch die Möglichkeiten der Philosophie angesichts dieser Gefahren genauer zu verstehen, ist es hilfreich, von einer kühnen Antwort auf die Motivationsfrage auszugehen. Diese kühne Antwort wäre, das philosophische Wissen sei bereits an sich ein Grund, es auch zu erlangen. Der Gedanke besteht darin, dass etwas zu wissen immer an sich gut sei und ein allgemeinstes Wissen zu haben eben allgemeinst oder umfassendst gut. Und weil es in diesem Sinn gut ist, hat man einen Grund, dieses Wissen zu erwerben. Man könnte das die Selbstbegründungsthese des philosophischen Wissens nennen. Danach ist es an sich begründet, dieses Wissen nachzufragen und gewinnen zu wollen. Allerdings ist die Selbstbegründungsthese unbefriedigend.

Die nächstliegende Möglichkeit, an der Selbstbegründungsthese zu zweifeln, setzt natürlich an der Annahme an, dass es ein solches allgemeines Wissen gibt und dass es von sich aus wertvoll ist. Die dabei zu überwindende Schwierigkeit ist unmittelbar wieder diejenige, die mit dem Einheitsproblem zu tun hat. Gibt es neben dem theoretischen Wissen auch ein praktisches, und wenn ja, wie hängen beide zusammen? Gibt es kein praktisches Wissen, wieso soll das theoretische Wissen wertvoll sein, wie kann es wertvoll sein, wenn es kein praktisches ist? Warum soll uns das allgemeinste Wissen interessieren, wenn wir es nicht zu einem bereits festliegenden Zweck verwenden, wie eben beim philosophischen Wissen?

Aus diesen Fragen ragt zunächst die heraus, ob man die Motivfrage zur Philosophie überhaupt repräsentativ über ein allgemeinstes Wissen angehen kann. Kann es ein solches allgemeinstes Wissen geben? Unter allgemeinstem Wissen kann man sich ein Wissen von ,allgemeinen Gegenständen“ vorstellen, im Unterschied zu wissenschaftlichem Wissen, etwa allgemeingültigen Gesetzen der Physik oder Chemie. Das allgemeinste Wissen ist dann nicht 
eine besondere, eben allgemeine Sorte des inhaltlichen Wissens, sondern ein Wissen von den Strukturen oder Voraussetzungen des inhaltlichen Wissens. Das allgemeinste Wissen ist ein Wissen von Gegenständen ganz allgemein, im diffusen, klärungsbedürftigen Sinn von „Gegenständen“, der Eigenschaften, Ereignisse, Materien, Beziehungen, Unterschiede, Ordnungen und deren Zusammenhänge einbezieht.

Weil das allgemeinste Wissen tendenziell ein Wissen vom Wissen ist, nennt man es auch häufig reflexives Wissen. Dabei erstreckt sich das Wissen nicht nur auf eines von den Eigenschaften der Gegenstände des Wissens, sondern auch auf das vom Wissen selbst, und vor allem auch auf das vom Wissenden. Zum allgemeinsten Wissen müsste auch ein Wissen vom Wissenden gehören, einmal, weil der Wissende zu den Gegenständen in der Welt gehört, zum andern aber, weil das Wissen ohne den Wissenden unmöglich ist. Warum die Antwort auf die Motivfrage in Form des allgemeinsten Wissens unzureichend ist - und warum sie den Unterschied zwischen theoretischem und praktischem Wissen nicht ausklammern darf -, zeigt sich an einem Folgeproblem dieser Reflexivität des allgemeinsten Wissens.

Wenn das allgemeinste Wissen ein Wissen von sich selbst umfasst, und das Wissen von sich selbst Gegenstand des Wissens wird, entsteht eine Lücke, in der der sich Wissende sich gleichzeitig deshalb nicht weiß, weil das Sich-Wissen bereits vom Sich-Wissen abhängt, und das Sich-Wissen nicht vollständig gegeben sein kann, wenn das Sich-Wissen des Sich-Wissenden eben nicht abgeschlossen ist. Damit zeigt sich, dass das allgemeinste Wissen, um das es der Philosophie geht, ein - je nachdem - Lücken-, Zirkel- oder Regressproblem hat. Der Einfachheit halber nenne ich es das Lückenproblem des Selbstwissens, als Teil des allgemeinsten Wissens. Eine Konsequenz dieses Problems scheint zu sein, dass die Haltung oder auch die Tätigkeit des Philosophen nicht einfach nur eine des Wissens sein kann. Im Wissen allein kriegt er sich gleichsam nicht zu fassen.

Eine weitere Konsequenz ist, dass die Motivation des Philosophen auf irgendeine Weise eine Reaktion auf das Lückenproblem, oder auch eine Kehrseite oder ein Bestandteil davon, sein muss. Diese Konsequenz ist deshalb beachtenswert, weil sich auf diese Weise eine systematische Verbindung zwischen dem durchschnittlichen Anspruch der Philosophie, nämlich Allgemeinwissen zu erhalten, und einer individuell zuschreibbaren - und mehr noch erfahrbaren - Haltung oder Problematik ergibt. Die Haltung der Philosophen sollte aus dem Zusammenhang des Lückenproblems heraus verständlich werden, denn was fehlt, was durch die Lücke ausgeblendet ist, ist ja gerade das Wissen von dem Individuum, das das allgemeine Wissen anstrebt. Was fehlt, ist also etwas Individuelles. Das auf eine selbst nicht wissensförmige Weise zu Ergänzende sollte dem nicht abschließbaren Wissen von sich selbst entsprechen.

Eine übliche Antwort an diesem Punkt ist, dass die Philosophie von einer Neugier oder einem Staunen ausgeht. Manche, wie Kevin Mulligan, reden auch von einem allgemeinen Interesse. ${ }^{1}$ Soweit es sich dabei um Beobachtungen handelt, sind sie nicht falsch, denn tatsächlich haben manche ohne weitere Erklärung ein solches Gefühl der Neugier, das sich auf das allgemeinste Wissen richtet - und dabei auch ein Wissen, das nicht bereits durch einen vorgegebenen Zweck motiviert ist. Wiederum bleibt die Sache unverständlich, wenn es nur bei diesem Konstatieren der Neugier bleibt, denn ob jemand die Neugier spürt, wäre dann ebenso willkürlich und zufällig wie die vorausgesetzten, noch nicht vom Wissen abhängigen Zwecke, auf Grund derer jemand ein bestimmtes Wissen interessant findet. Man hätte dann noch keine Erklärung, und keine Begründung, der speziellen ,philosophischen Neugier“.

1 K. Mulligan, Torheit, Vernünftigkeit und der Wert des Wissens, in: G. Schönrich (Hg.), Wissen und Werte, Paderborn 2009, 27-44. 
Meines Erachtens zeigt sich an diesem Punkt bereits eine typische Schwierigkeit, in die sich das philosophische Reden selbst bringt, indem es sich auf einen einzigen, isoliert gehandhabten Begriff bezieht und nur noch versucht, eine Antwort im Rahmen seiner begrenzten Mittel zu finden. Das mysteriöse Staunen ist eine Pseudoerklärung für das Wissen an sich, wenn es vom zweckrationalen Wissen völlig getrennt worden ist. Die Alternative wäre, nicht einen Gegensatz zwischen zweckrationalem Wissen und Wissen an sich zu bilden, sondern das zweckrationale Wissen selbst möglichst allgemein zu fassen.

Dieser Befund deckt sich auch mit der Beobachtung der Wissenslücke, die nur durch eine praktische Haltung geschlossen werden kann. Daraus sollte man folgern, dass es sich beim philosophischen Wissen um ein praktisches Wissen, oder ein Wissen auf praktischer Grundlage, handelt, sodass die kognitive Existenz nur eine Teilrolle spielt. Wovon könnte das praktische Wissen aber ein Wissen sein? Das zweckrationale Wissen möglichst umfassend verstanden, wäre es ein Wissen von der individuellen Lebenssituation, in der sich jeder von uns befindet, und das wiederum allgemein verstanden. Die knappste Erklärung des berühmten Staunens könnte sein, dass man sich des Kontrasts zwischen dem Allgemeinen in der Welt und sich selbst als Individuum bewusst wird, und sich fragt, wie man zu diesem Allgemeinen, oder auch zu allem anderen, passt. Ursula Wolf hat in einem Buch, dessen historischen Skizzen ich gleich ein Stück folgen werde, den praktischen Anstoß für die Philosophie durch die Aporien zu erklären versucht, in die jeder in seinem individuellen Leben praktisch gerät. ${ }^{2}$ Wolf geht so weit, die einzelnen Phasen der Geschichte der Philosophie durch transformierte und teilweise verdrängte existenzielle Probleme erklären $\mathrm{zu}$ wollen. Wahrscheinlich sind die Selbstverwicklungen der Philosophie insgesamt weniger systematisch zu erklären und verdanken sich Großteils einfach der Möglichkeit, von praktischen Kontexten abgehobene Gedanken entwickeln zu können. Dennoch ist die Annahme erhellend, dass die Philosophie grundsätzlich von der Ethik ausgeht und dort Schiffbruch erleidet, wo ihr dieser Ausgang weitgehend undurchsichtig geworden ist - dass ihr also dort ihre innere Einheit zu einem unlösbaren Problem wird, wo sie vergisst, dass einzig die Ethik das Bedürfnis erklärt, eine Einheit - nämlich eine Einheit im individuellen und sozialen Leben - als solche anzustreben. Eine skizzenhafte, in drei Modelle gegliederte Geschichte der Philosophie scheint das zu belegen.

\section{Modell 1 - kritische Selbstprüfung: Sokrates' Vorbild, Philosophie als Ethik}

Das sokratische Modell der Philosophie ist das paradigmatische Modell der kritisch-ethischen Selbstprüfung. Die Rede von einem „Modell“ ist wegen ihrer Nähe zu einer (geschlossenen) Theorie leicht irreführend, soll aber die Übertragbarkeit dieser Auffassung betonen. Kritisch ist die Selbstprüfung, weil in ihr eine Skepsis gegenüber dem konventionellen Meinen in der Gesellschaft zum Ausdruck kommt. Ethisch ist sie, insofern es um die Beschaffenheit des ganzen Lebens geht. Selbstprüfung ist die Selbstprüfung in dem Sinn, dass es vorrangig und ursprünglich um eine Beschäftigung mit der individuellen Person geht, nicht um ein Prüfen von externen Tatsachen oder Wahrheiten. Instabil und vorläufig ist diese Selbstprüfung, insofern sie keine zufriedenstellende Antwort erhält. Wichtig für uns Heutige ist diese Selbstprüfung, weil wir angesichts der historisch an sie anschließenden, aber problematisch

2 U. Wolf, Die Philosophie und die Frage nach dem guten Leben, Reinbek bei Hamburg 1999. 
bleibenden Versuche nach Sokrates auf sie zurückverwiesen sind. Im Kontrast zwischen dem sokratischen Ausgangspunkt der Philosophie und der Geschichte von weiteren Antworten und Vorschlägen befinden wir uns wieder erstaunlich nah am Ausgangspunkt. ${ }^{3}$

Verkörpert wird das kritische Selbstprüfen durch den historischen Sokrates. Dieser Sokrates ist sichtbar im Kontrast zu den griechischen Naturphilosophen, den Sophisten und dem platonischen Sokrates. Die Naturphilosophen sind voraufklärerische Metaphysiker, deren globale Theorien bestenfalls Ideenanstöße geben. Die Sophisten sind Sozialtechniker, die sich der Begründungsproblematik bewusst sind, sich ihr aber auch bewusst verweigern und einen direkt erfolgsorientierten Pragmatismus vertreten. Der platonische Sokrates glaubt, im Unterschied zum historischen, ein allgemeines Wissen vom Guten und dessen Erkennbarkeit erreichen oder besitzen zu können. Historisch gesehen, im Rahmen der westlichen Philosophie, steht der platonische Sokrates bereits für die zweite, die wissenschaftliche Phase der Philosophie, während der historische für die erste, lebenspraktische Phase steht. Im Folgenden meine ich mit „Sokrates“ immer den historischen der ersten Phase, und das Modell der kritischen Selbstprüfung kann mit diesem Sokrates identifiziert und veranschaulicht werden.

Die Figur des Sokrates in den platonischen Frühdialogen veranschaulicht mehrerlei. Erstens und vordringlich die Haltung der persönlichen Selbstprüfung, wie sie vermutlich am klarsten in der Behauptung der Apologie zum Ausdruck kommt, wonach ein Leben ohne Selbsterforschung gar nicht „verdient, gelebt zu werden“ (38a). Im selben Zusammenhang hebt Sokrates hervor, dass sich über die Tugenden zu unterhalten das größte Gut des Menschen sei, die Selbsterforschung hat also eine explizit ethische Ausrichtung.

Zweitens ist Anlass für diese Selbstprüfung die Erfahrung des Zufalls und der schicksalshaften Umstände des Lebens, in denen sich eine Lebensordnung nicht von selbst ergibt, sondern aktiv hergestellt werden muss. Diese Aktivität verweist auf die Tugenden als Qualitäten des eigenen Handelns. Im Kontrast sind die kritisierten Gesprächspartner des Sokrates immer solche, die sich ihrer Passivität und Ausgeliefertheit an die Umstände entweder nicht bewusst sind oder sie aus einem Selbstirrtum heraus bejahen oder hochschätzen. Die rein Naiven sind eher die Sänger und Schauspieler, die raffiniert Verwirrten sind durchweg die Sophisten. Der Rapsode Ion glaubt, dass er als Sprachrohr Homers alle von Homer beschriebenen Fähigkeiten besitzt. Einer der Schüler des Sokrates, Krito, begründet den Versuch, ihm zur Flucht verhelfen zu wollen, mit seiner Furcht vor der Meinung der anderen, sollte er den Freundschaftsdienst versagen. Die Sophisten Gorgias und Polus vertreten den Maßstab des sozialen Erfolgs, ohne erklären zu können, wofür sie darüber hinaus eigentlich selbst einstehen.

Drittens - und insgesamt am wichtigsten - bleibt die von Sokrates vertretene Art des Selbstwissens auf eine charakteristische Weise hypothetisch. Auf der einen Seite ist die Fragetechnik und Gesprächsführung klar von einer metaphysisch ausgerichteten Definitionsidee geleitet, indem das Gespräch immer darauf abzielt, häufig auch damit beginnt, eine Wesens- und Realdefinition von etwas zu erlangen, in der Regel einer Tugend. Durch die auf ein Wesen zielende Definitionsfrage ist es für Sokrates leicht, das konventionelle Wissen der Gesprächspartner zu erschüttern. Auf der anderen Seite wird einer positiven Antwort oder einem Wesen der Tugenden nicht vertraut und das Bekenntnis von Sokrates (in der Apologie), einzig zu wissen, dass er nichts wisse, ist in diesem Sinn des mangelnden Verfügens über letzte Sicherheit kennzeichnend.

3 Das ist zumindest eine herrschende Meinung unter vielen praktischen Philosophen, weniger natürlich unter den theoretischen. Deren aktuelle Professionalität anhand einer Unzahl von Teilthemen hat in der Antike kein Vorbild. 
Instabil und offen bleibt die damit verbundene Gesprächstechnik insofern, als mangels sicherem Definitionswissen oder gar der aufscheinenden prinzipiellen Unmöglichkeit solchen Wissens auch die Kritik an den weniger reflektierten Partnern nicht mehr völlig überzeugt, insbesondere nicht an den Sophisten, die sich im Licht dieses Versagens mit guten Gründen zu weigern scheinen, Sokrates auf dem Weg zu den Wesensdefinitionen zu folgen. Sokrates scheint zwar reflektierter als die Sophisten, aber deren Position scheint am Ende aus Gründen der praktischen Notwendigkeit heraus mindestens erwägenswert, weil praktisch vorteilhaft, wenn nicht für ein durchschnittliches Leben sogar notwendig.

Man kann diese sokratische Position auch so zusammenfassen, dass sie Ausdruck einer praktischen Aporie ist. Im Vergleich zu den Sophisten scheint klar, dass ein das Leben vereinheitlichendes Wissen vom Guten erstrebenswert ist, im Vergleich zu den gegenüber Sokrates früheren und späteren Metaphysikern ist aber auch klar, dass ein solches Wissen nicht zur Verfügung steht. Es ist nicht so, dass Sokrates daraus eine theoretische oder sonst wie tiefere Lehre zu ziehen scheint, außer eben der konkret praktischen, wonach die fortwährende Selbstprüfung zum Lebensinhalt geworden ist. Diese Position wird aber selbst nicht verteidigt, und sie ist, wie eben angedeutet, darin problematisch, dass ihr Sinn mangels realisierbarem Ziel auch bestritten werden kann.

Vermutlich ist die schwere Akzeptierbarkeit dieser praktischen Aporie, wie auch die alltäglich-praktische Unmöglichkeit für die meisten Menschen, ein sokratisches Leben zu führen, die Erklärung der beiden nach Sokrates folgenden philosophischen Entwicklungen. Platon macht schon im Staat deutlich, dass nicht alle Menschen ein sokratisches Leben führen können, indem er neben den Philosophen noch die Arbeiter und Soldaten vorsieht, die gerade nicht das Leben der Philosophen leben. Seine Philosophen unterscheiden sich von Sokrates darin, dass sie zumindest ansatzweise über metaphysisches, also nicht-empirisches Wissen verfügen, mit dessen Hilfe sie auch den Staat und die sozialen Umstände für die meisten anderen Menschen gestalten können sollen. Die Ethik ist nach wie vor die dominierende und extensive Konzeption der Philosophie generell, auch wenn bestimmte theoretische Reflexionen bei Platon ein zunehmend größeres Gewicht annehmen als bei Sokrates. Sokrates allerdings hätte das platonische Wissen und die damit verbundenen philosophischen Theorien nicht akzeptiert.

Die zweite, folgenreichere Entwicklung nach Sokrates besteht in der aristotelischen Trennung von Ethik und Metaphysik. Aporien werden von Aristoteles zwar anerkannt, bleiben aber als Thematik auf die Metaphysik beschränkt und haben keinen Einfluss mehr auf die Ethik und Politik. Die Ethik schildert ein im Prinzip einheitlich realisierbares, gezielt ausgerichtetes Leben der Tugenden, dessen Inhalt im Wesentlichen in der Perfektion der Tugenden und den beiden Hauptzielen theoretisches Wissen und Politik besteht, mit einer leichten Bevorzugung des letzteren Ziels der Politik, weil das theoretische Wissen göttlich ist und damit von Menschen nicht wirklich realisierbar.

Auch diese Ausrichtung auf die Politik ist ein Teil des Versuchs, das praktische Leben von den prinzipiellen Problemen abzukoppeln und die unlösbaren Aporien in ihrer Wirkung auf das praktische Leben gewissermaßen abzuschirmen. Mit Aristoteles beginnt die bis heute einflussreiche und für die westliche Philosophie repräsentative Trennung von theoretischer und praktischer Philosophie, die auch bedeutet, dass die praktische Philosophie der harmlosere, weniger gewichtige Teil der Philosophie wird. In manchen Teilen der Philosophie, insbesondere bei Hegel und Marx, hatte das nicht nur innerphilosophisch fatale Folgen. Nicht nur deshalb, sondern aus internen Gründen zeigt sich im Weiteren, dass sich die Trennung und der Vorrang der „ersten“, theoretischen Philosophie nicht halten lässt, und dass wir auch in diesem Punkt wieder auf Sokrates zurückkommen müssen. 


\section{Modell 2 - Erkenntnistheorie: Philosophie als Wissenschaft}

Am wirkungsmächtigsten gilt die Trennung zwischen theoretischer und praktischer Vernunft für Kant. Die ersten Sätze des Vorworts zur ersten Auflage der Kritik der reinen Vernunft machen sowohl deutlich, dass Kant diese Trennung vollzieht, als auch, warum er sich so etwas wie ein tieferes Motivationsproblem der Philosophie, oder gar ein Entspringen der Philosophie aus praktischen Schwierigkeiten, schwer vorstellen kann:

„Die menschliche Vernunft hat das besondre Schicksal in einer Gattung ihrer Erkenntnisse: dass sie durch Fragen belästigt wird, die sie nicht abweisen kann, denn sie sind ihr durch die Natur der Vernunft selbst aufgegeben, die sie aber auch nicht beantworten kann, denn sie übersteigen alles Vermögen der menschlichen Vernunft.“ (KrV, AVII)

Nach der in diesen Sätzen enthaltenen Vorstellung von Philosophie ist der Gegenstand der Philosophie erstens vorrangig die Vernunft, zweitens ist die Vernunft ein objektives Geflecht von Handlungen und Schlüssen, und drittens ist sie antinomisch, sie verwickelt sich in Widersprüche. Wenn man fragt, warum jemand an Philosophie, sprich: an der Analyse der und Reaktion auf die Eigenarten der Vernunft, interessiert sein sollte, ist die Antwort eben, dass Menschen kraft ihrer Teilhabe an der Vernunft notwendig motiviert sind, sich für ihre Eigenschaften zu interessieren. Die Fragen nach den drei metaphysischen Zentralideen Gott, Freiheit und Unsterblichkeit, nach Kant die antinomischen Themen der Vernunft, sind also solche, an denen vernünftige Wesen notwendig teilhaben und in deren Widersprüche sie verwickelt sind.

Plausibel ist an diesem Gedankengang, dass Menschen kraft ihrer Reflexivität in bestimmte Probleme hineingetrieben werden, und dass ein Merkmal dieser Probleme ihre Antinomieanfälligkeit ist. Worin sich Kant mit Aristoteles tendenziell trifft, ist die theoretische Form der Antinomien, während es für Sokrates ein Problem war, dass das konkrete praktische Leben nicht einheitlich und ganzheitlich bestimmt war. Kant sieht das Hauptproblem der Metaphysik in der Unendlichkeit der Begriffe, was als ein praktisches Problem schwer vorstellbar ist. Die Antinomien der Vernunft sind deshalb vorrangig solche, die für Kant im spekulativen, nicht im praktischen „Gebrauch der Vernunft" auftreten. ${ }^{4}$

Ähnlich wie für Aristoteles sind damit für Kant die prinzipiellen Herausforderungen an unser Lebens- und Weltverständnis auf die theoretische Ebene beschränkt und werden von der praktischen säuberlich ferngehalten. Moralische Dilemmata oder tiefgreifende Konflikte im praktischen Leben gibt es für Kant nicht, auch wenn in seiner Ethik eine Art Kasuistik vorgesehen ist. Die Kasuistik ist eine sekundäre Anwendungsdisziplin (Anwendung des vernunftgegebenen kategorischen Imperativs), die Ethik entspringt nicht der Erfahrung von praktischen Konflikten und Aporien. Selbst für die Idee der Freiheit als derjenigen der drei genannten Ideen, die den Knotenpunkt zwischen dem theoretischen und praktischen Bereich bildet, wird zwischen dem theoretischen und dem praktischen „Gebrauch“ dieser Idee unterschieden. Die Aporetik bei dieser Idee, der Konflikt zwischen Determinismus und Willensfreiheit, soll auf die theoretische Seite der Idee beschränkt sein. Für den praktischen Gebrauch

4 Kant konstruiert zwar in der Kritik der praktischen Vernunft die „praktische Antinomie“ von Neigung und Sollen, und in der Religionsschrift eine Antinomie des Glaubens aus Gott und aus der Moral, aber diese Antinomien sind nicht mehr bedeutsam für die ihm vorrangig wichtigen „Grenzen der Vernunft". 
vertritt Kant einen Kompatibilismus, nämlich die These, dass der Determinismus kein Hindernis für praktische Freiheit wird, weil das moralische Handeln vollständiger Ausdruck der Freiheit ist und es im Prinzip die Kontrolle über die Sinnlichkeit behält. ${ }^{5}$

Die kantischen Menschen haben mit den aristotelischen gemeinsam, und unterscheiden sich darin von Sokrates, dass sie ein im Wesentlichen konfliktfreies Leben führen können. Stärker noch als bei Aristoteles sind ihre Handlungen durch Regeln gebunden, und viel einfacher als bei ihm ist ihr innerer Konflikt einzig der einer rationalen Selbstkontrolle, während die aristotelischen Akteure ihre persönlichen Qualitäten in ihrer Vielfalt ausbilden und darin individuell sein können. Der ideale kantische Akteur würde, wenn es ihn gäbe, alle Individualität verlieren. Viel interessanter und gegenwartsnäher scheint demgegenüber Sokrates darin, dass er sich und die anderen zu einem Gespräch auffordert, zu dessen Zielen er selbst noch keine Antwort hat.

Angesichts der Einseitigkeit des historischen Kant haben heutige Kantianerinnen modernisierende Lektüren angesetzt, mit denen der praktische Gebrauch der Vernunft aufgewertet würde. So hat Christine Korsgaard den Kategorischen Imperativ als einen Haltepunkt für ein persönliches Identitätsproblem gelesen, wodurch eine kartesianische Problematik in praktische Subjektsicherheit überführt würde. ${ }^{6}$ Onora O'Neill hat den bei Kant vage bleibenden Zusammenhang von theoretischem und praktischem Vernunftgebrauch so präzisiert, dass das moralische Gebot auch das Erkennen in ein intersubjektives Begründen gegenüber anderen transformiert. ${ }^{7}$ Diese „kantianischen“, nicht mehr kantischen Figuren bleiben dem Verdacht ausgesetzt, dass sie das vorgesetzte Problem - hier moralische Identität, da empirische Wahrheit - nicht wirklich lösen können. Problematischer halten sie aber an der zentralen Prämisse fest, dass es eine historisch nicht relative moralische Grundevidenz gibt, anhand derer die praktische Vernunft identifiziert werden kann.

Eine radikale Selbstvergewisserung der Moral ist für Kant unnötig, weil er sich vermutlich ein Außerkrafttreten der Moral, einen gelebten Amoralismus, gar nicht vorstellen konnte. Als Theoretiker konnte er ihn sich nicht vorstellen, weil er sich die Abwesenheit der Vernunft nicht vorstellen konnte. Wenn es die Vernunft gibt, werden alle praktizierten Amoralismen als Defizite erkennbar und man kann und muss am Ideal gegenüber der mangelhaften Welt festhalten. Klammert man jedoch alle problematischen Versuche des nicht-empirischen Erkennens der Vernunft, also einer Metaphysik der Vernunft, aus, so bliebe nur die Begründung, wonach die Unterstellung der Vernunft nötig ist, um die Welt so zu erhalten, wie sie tatsächlich zu sein scheint. Was die Moral betrifft, ist die reale Welt aber nicht moralisch geordnet, und ob wir uns eine durchgängige moralische Ordnung in der Realität überhaupt wünschen sollten, ist zweifelhaft. Die Moral und die Vernunft in Form einer transzendentalen Voraussetzung dessen zu gewinnen, was man nicht aufgeben kann, ist also ebenfalls nicht überzeugend.

Welches könnten die praktischen Probleme sein, die hinter dem kantischen Bemühen um die Lösung der Antinomien, oder hinter dem kartesianischen Streben nach Gewissheit liegen? Dem Descartesschen Ringen mit dem Zweifel, ob man selbst existiert und überhaupt irgendwelche Bewusstseinsinhalte hat, liegt offenkundig eine spielerisch erweiterte Unsicherheit

5 A. Wood, Kant's Compatibilism, in: ders. (Hg.), Self and Nature in Kant's Philosophy, Ithaca 1984, 73-101.

6 Ch. Korsgaard, The Sources of Normativity, Cambridge 1996; dies., Self-Constitution, Oxford 2009.

7 O. O'Neill, Reason and Politics in the Kantian Enterprise, in: dies., Construction of Reasons, Cambridge 1989; dies., Vindicating Reason, in: P. Guyer (Hg.), The Cambridge Companion to Kant, Cambridge 1992. 
oder Orientierungslosigkeit zu Grunde. Man kann sich vorstellen, dass man sich in der fremden Stadt verläuft und nicht mehr weiß, wo man sich befindet. Und weiter getrieben kann man sich auch vorstellen, dass man nicht mehr weiß, wer man ist, wo und wann man lebt etc. In gewisser Weise ist Kant ebenfalls von der methodisch oder theoretisch erweiterten Zweifelsproblematik befallen, insofern die metaphysischen Ideen ja solche sind, die unendlich sind und sich ihrer Unendlichkeit wegen, die letztlich doch nur noch zeitlich vorgestellt werden kann, der Erkenntnis entziehen.

Wie beim spielerischen Wissenszweifel wird auch bei Kants unendlichen Reihen sichtbar, dass die Trennung zwischen theoretischer und praktischer Reflexion die Hauptursache für die Problemformulierung ist. Bliebe die theoretische Erkenntnis an die praktische rückgebunden, würde das unendliche Wissen allererst kein Problem, die angebliche Lösung der „regulativen Ideen“ würde schon von Beginn an wirksam. Bei Descartes' Zweifelsproblematik würde die praktische Seite mit zu berücksichtigen bedeuten, dass der Akteur anhaltend unter einem Handlungszwang steht, sich ernähren, kleiden, bewegen muss, und deshalb praktisch genötigt ist, einen, wie Charles Peirce angemerkt hat, „lebendigen“ vom „papiernen“ Zweifel zu unterscheiden. ${ }^{8}$ Zweifelsgründe, die ihm das Leben von vornherein unmöglich machen, scheiden dann als „gute Gründe“ aus. Das praktische Subjekt hat andere Kriterien für Zweifel als das isoliert-theoretische, und nur durch die Trennung beider entsteht überhaupt das Bedürfnis nach einer gesonderten theoretischen Philosophie.

\section{Modell 3 - Lebensaspekte: Philosophie als Anthropologie}

Nach meiner bisherigen Schilderung hat sich die Einheitsfrage beim historischen Sokrates als lebenspraktische Frage etabliert und ist dann über Platon und Aristoteles, Descartes und Kant zu einer durch die interne Teilung multiplizierten eigenständigen Problematik geworden. Kant muss sich je die Einheit der Erkenntnis und der Moral, sowie zusätzlich der Erkenntnis und der Moral in einer Vernunft, als Probleme vorsetzen, und da er das Einheitsproblem im ersten Schritt nur auf der Höhe der Metaphysik durchspielt, vermag er nachträglich eine überzeugende Verbindung mit der im konkreten Leben (Sinnlichkeit) agierenden praktischen Vernunft nicht mehr herzustellen. Zwar bleibt, wie der knappe Abschnitt vom „Primat der reinen praktischen Vernunft“ $(\mathrm{KpV})$ sagt, die spekulative Vernunft in ihren regulativen Prinzipien von der praktischen abhängig, aber erstens nur völlig formal und zweitens nur im abstrakten Sinn der praktischen Vernunft. Da auch das Glück im „höchsten Gut“ zwar formal integriert wird, scheint auf dem Papier eine Einheit hergestellt - aber das kantische Glück hat mit dem realen Glück wenig zu tun, es ist (wie auch die kantische Religion) ein Glück von Gnaden der Moral.

Angesichts dieses Ergebnisses einer formalen zu Lasten einer realistischen, lebenspraktischen Einheit ist verständlich, dass sich eine ,anthropologische“ Strömung in der Philosophie entwickeln musste, die nun versuchte, die Verhältnisse zu Gunsten des Primats des Praktischen grundsätzlich umzukehren. ${ }^{9}$ Je nachdem, wie eng oder weit, wie implizit oder explizit

8 Ch. S. Peirce, The Fixation of Belief [1877], in: The Essential Peirce, Bd. 1 (1867-1893), hg. v. N. Houser u. C. Kloesel, Bloomington 1992.

9 Der Begriff der Anthropologie hat, ausgehend von Kants Gebrauch, einen überwiegend wissenschaftlichen Klang. Heidegger, den ich auf dieser dritten Stufe einordnen möchte, hat sich in Sein und Zeit gegenüber der Anthropologie deshalb bewusst abgegrenzt. 
man die Bedingung der Lebensthematik nimmt, wird man eine unterschiedlich große Zahl von Philosophen des 19. und 20. Jahrhunderts unter diesem Modell vertreten sehen. Klar scheint, dass Nietzsche, Dilthey und Heidegger drei vorrangige Vertreter einer solchen anthropologischen Position sind, erkennbar am dionysischen Bedürfnis nach Einheit und Ganzheit bei Nietzsche, der Orientierungslosigkeit im Sinnganzen bei Dilthey und der Erfahrung der existenziellen Angst bei Heidegger. ${ }^{10}$

Auf abgeschwächte Weise finden sich zu Dilthey und Heidegger auch Analogien und Parallelmotivationen im Bereich der analytischen Philosophie, auch wenn die analytische Philosophie - auf Grund der in ihr ebenfalls aufrechterhaltenen theoretischen Tradition - zu Teilen im Stil der klassischen Erkenntnistheorie und Metaphysik betrieben wird. Die zentrale ,,analytische Aufgabe“, bestimmte Begriffszusammenhänge zu klären, kann aus verschiedenen Motiven heraus betrieben werden: entweder (praktisch), weil ein aporetisch angestoßenes Orientierungsbedürfnis vorliegt, oder (theoretisch) weil man es als eine sich selbst erklärende Aufgabe ansieht, etwa unter Berufung auf Klarheit als selbsterklärendem Wert. Einen Rückschritt gegenüber Kant (und mehr noch Hegel) bedeutet die einfache Begriffsanalyse darin, dass der Anstoß durch Antinomien auch im rein theoretischen Bereich nicht mehr explizit vorhanden ist. Begriffe werden geklärt, weil Begriffe isoliert betrachtet meist unklar werden, nicht, weil sie klar und widersprüchlich sind. Der Metaphysikverdacht des Neopositivismus hat sich in ein pauschal gewordenes Klärungsbedürfnis transformiert, das sich durch Abstraktion einerseits gleichsam selbst erschafft, andererseits ohne Blick auf ein Wozu ziellos bleibt. Das grenzenlose Bedürfnis nach Klarheit entsteht aus der Unklarheit über das Bedürfnis.

Die verschiedenen Versionen der Gegenwartsphilosophie zusammenfassend, könnte man auch von einer anthropologischen und einer verkürzten Hermeneutik sprechen. Beiden ist gemeinsam, dass sie den Menschen im Zusammenhang seiner Sinnsysteme wie Sprachen, Kulturen, Gesellschaften, Traditionen, Handlungssituationen verstehen wollen und dadurch, dass sie diesen auf Einzelsituationen bezogenen Verstehensbegriff benutzen, die übliche Unterscheidung zwischen einem theoretischen, auf Erkenntnis zielenden und einem praktischen, dem Handeln gewidmeten Bereich vermeiden. Zwischen beiden Bereichen zu unterscheiden, ist kein Programm mehr, die Unterscheidung ist keine der Bereiche und Zuständigkeiten. Die anthropologische Hermeneutik unterscheidet sich von der verkürzten allerdings dadurch, dass sie sich aus einem Bewusstsein der existenziellen Probleme heraus entwickelt, während die verkürzte Hermeneutik, eben große Teile der analytischen Philosophie, ein solches Bewusstsein für unnötig oder unmöglich hält. ${ }^{11}$

Eine dritte Unterposition dieses anthropologischen Modells, die irgendwie zwischen den beiden genannten steht, ist der Pragmatismus von James und Dewey. Charakteristisch ist für ihn, dass er die vielleicht knappste, aber in dieser Knappheit problematische Version der anthropologischen Hermeneutik ist. Charakteristisch ist für diesen Pragmatismus der Instrumentalismus, also die Ansicht, dass alles typisch Menschliche eine Form instrumentellen Handelns ist und dass das instrumentelle Handeln im Wesentlichen biologisch als Lebensbewältigung verstanden werden kann. Anthropologisch ist dieser Gedanke insofern, als er von

10 Hier folge ich den treffenden Einzelanalysen bei: U. Wolf, Die Philosophie und die Frage nach dem guten Leben, a. a. O.

11 Wittgenstein ist als der berühmte Verschweiger der praktischen Seite bekannt, in letzter Zeit sind es vor allem Stanley Cavell und Harry Frankfurt, die damit begonnen haben, der angelsächsischen Philosophie wieder die Augen für existenzielle Voraussetzungen zu öffnen. Donald Davidson wäre hingegen ein exzellenter Vertreter der verkürzten Hermeneutik, weil seine Handlungstheorie keine kreative Rolle für den Bedeutungsholismus spielt, und ebenso wenig umgekehrt. 
biologischen Zwängen und Problemen ausgeht, wie Hunger und Unsicherheit, und zu einer radikal praktischen Position führt er deshalb, weil eben auch alles theoretische Denken als Form des Handelns aufgefasst wird, das auf Lebensziele hin ausgerichtet ist. Das pragmatistische Individuum ist in einem trial-and-error-Prozess auf seine Überlebensziele hin bezogen. Eine ebenfalls verkürzte Hermeneutik ergibt sich dann deshalb, weil die Folgen dessen nicht mitbeachtet werden, dass für Menschen Überlebensziele allein nicht ausreichen, sondern dass Menschen qualifizierte Ziele benötigen, die über die biologische Notwendigkeit hinausgehen.

Doch es ist einfach, den Pragmatismus in diesem Punkt zu kritisieren, welche anderen Möglichkeiten für einen Umgang mit Handlungszielen gibt es? Wir sind jetzt wieder an dem Punkt angelangt, an dem auch Sokrates stand, oder steht, wenn er zwar einerseits die Sophisten für besiegt erklärt, aber selbst nicht sagen kann, welchen Tugenden man eigentlich folgen soll. Welche existenziellen Probleme und Anstöße sind es, oder könnten es sein, derer die anthropologische Hermeneutik sich bewusst ist? Nimmt man das Verstehen seiner selbst in seiner Situation als die vordringliche philosophische Aufgabe, dann drängen sich vor allem zwei Teilprobleme auf, einmal das Verstehen seiner selbst im eigenen Leben und zum andern das Verstehen seiner selbst in Gegensatz oder in Verbindung zu den anderen Mitmenschen mit anderen Worten, das biographische und das soziale Verstehen. Das Verstehen seiner selbst auf dem Hintergrund der Geschichte, der Zukunft, des Kosmos, der nichtmenschlichen Intelligenz sind weitere Verstehensprobleme, die aber weniger grundsätzlich zu sein scheinen wie das biographische und das soziale Verstehen.

Dem biographischen und sozialen Verstehen lassen sich je typische Lebens- oder Existenzprobleme zuordnen. Das vordringliche biographische Lebensproblem ist vermutlich das der Endlichkeit des eigenen Lebens, das vordringliche soziale Lebensproblem ist vermutlich das der sozialen Trennung und der unaufhebbaren Fremdheit der anderen. Beide Lebensprobleme liegen einer Fülle von bekannten alltäglichen Problemen zu Grunde. In das biographische Problem fällt der Umgang mit dem Tod, dem je anderen wie dem eigenen, aber auch dessen vorweggenommene Bedeutung für das durchschnittliche Leben. Ohne den Tod keine Lebensperioden, kein Erfolg oder Misserfolg von Lebensprojekten, kein Wert von Aufgaben und Handlungen, kein Risiko. Nicht nur ist der Tod also indirekt an vielem beteiligt, was wir als festen Bestandteil des Lebens nehmen, er wirft fortwährend die Frage auf, ob auch die Teilprojekte, die uns möglich sind, innerhalb des Lebens sinnvoll sind. Er wirft sie insbesondere dann auf, wenn man wie Heidegger der Meinung ist, dass der Tod das einzige Ziel des Lebens ist, in dessen Bewusstsein wir „eigentlich“ handeln sollten. In das soziale Problem fallen alle konflikthaften sozialen Beziehungen, von sprachlichen und kulturellen Differenzen bis zu Vertrauensbrüchen, gescheiterten Ehen, Gewalttaten und Kriegen. Aber auch die anhaltende Schwierigkeit, zu sagen, wieweit wir anderen gegenüber frei sind oder sein sollen, ob Autonomie eine sinnvolle Vorstellung ist, ob es Nationalstaaten geben soll oder nur noch Märkte und Versicherungsverbände.

Gibt es, könnte man fragen, einen untergründigen „Motor“ für das soziale Problem? Am ehesten den der gleichzeitigen Notwendigkeit von Freiheit und Abhängigkeit. Um Individuen zu sein, benötigen wir Freiheit, aber zugleich die anderen zu den verschiedensten Zwecken der Individualisierung und Gemeinschaft. Beides ist nötig, aber nicht gleichzeitig möglich. Ähnlich liegt der „Motor“ für das biographische Problem vermutlich in der gleichzeitigen Anforderung, erfolgreich zu sein und zu scheitern, handeln zu können und zu erleiden, oder ins Extrem gedacht, unendlich zu leben und sterblich zu sein. Erfolgreich sein könnte man nicht, wenn Scheitern nicht vorgesehen wäre. Der eigene Erfolg würde nichts bedeuten, wenn er nicht vom Scheitern begleitet wäre. Handeln könnte man nicht, wenn man nicht ebenso Opfer wäre. Projekte mit einem Risiko realisieren wäre unmöglich, wenn man nicht sterben müsste. 
Bevor ich hieraus abschließend Folgerungen für die Einheitsfrage zu ziehen versuche, fasse ich jetzt diesen Überblick noch einmal zusammen. Die übergreifende Frage war, ob die Philosophie eine Einheit hat, und soweit ja, woher sich der Zwang zu einer Einheit ergibt. Die am historischen Sokrates veranschaulichte These war (inspiriert durch Ursula Wolf), dass sich das Bedürfnis nach einer Einheit zunächst an den durchschnittlichen Lebenskonflikten entzündet und von da in die Philosophie einwandert. Die Philosophie reagiert auf die Lebenskonflikte, entwickelt aber zugleich eine Metaphysik, in der sich die praktischen Lebenskonflikte in theoretische Aporien verwandeln. Indem sie zwischen theoretischer und praktischer Philosophie trennt (Aristoteles, Kant), reagiert die Philosophie wiederum auf die praktischen Erfordernisse und isoliert die theoretischen Aporien gegenüber dem Leben. Dadurch multiplizieren sich, wie bei Kant, Meta-Einheits- und Legitimationsprobleme. Aber nicht nur intern, sondern auch von außen gesehen bedeutet das Teilen in der Philosophie, sich der Motivation zu berauben, eine Antwort auf die Motivfrage der Studenten zu finden. Deshalb ist der einzige Weg, auf die Ganzheitsbedürfnisse und Einheitsbedrohungen vor der Philosophie, die des individuellen und sozialen Lebens, zurückzugehen und die Aporien der Philosophie von ihnen her teils zu korrigieren, teils neu zu fokussieren. Auf unterschiedliche Weise war das bereits die Methode der anthropologischen Philosophen.

\section{Quietisten und Konstruktivisten}

Die Frage drängt sich auf, warum die anthropologischen Philosophen nicht hinreichend erfolgreich waren, die Philosophie insgesamt unter den Primat des Praktischen zu stellen. Eine versöhnliche Antwort wäre, dass ihnen das schon gelungen ist, die relative Eigenständigkeit der theoretischen Fragen im Einzelnen nur leicht darüber hinwegtäuscht. Ich glaube, dass diese Antwort irreführend ist und das Weiterbestehen der isoliert theoretischen Philosophie sich eher der Unklarheit der Alternative verdankt. Die Unklarheit besteht nicht nur dazu, welches die Alternative ist, sondern radikaler darin, ob es eine Alternative überhaupt gibt. Die verschiedenen anthropologischen Philosophen illustrieren verschiedenartige praktische Aporien, und selbst wenn man von den genannten Aporien der individuellen und sozialen Existenz ausgeht, wie soll man diese Aporien bearbeiten, wenn nicht theoretisch?

Um die Möglichkeiten der „praktischen Alternative“, also des Versuchs der Einheit der Philosophie von der Lebens- und Sozialpraxis her, überschaubar werden zu lassen, ist es hilfreich, zwischen einer quietistischen und einer konstruktiven Position zu unterscheiden. Die quietistische Position ergibt sich aus dem Versuch, die philosophischen Probleme so eng wie möglich an die Alltagsbedürfnisse zu binden. Die traditionellen Quietisten waren die hellenistischen Stoiker. Indem sie die Konflikte zwischen ihren Lebenswünschen und den nicht erkennbaren Lebenszielen so zu lösen versuchten, dass sie die Lebenswünsche bestritten oder sich abzugewöhnen suchten, entfiel das Problem, wie die individuelle Existenz in das Allgemeine passt, und damit auch die typische Motivation für die Philosophie. Lebensweisheit entsteht dann im Sinn eines quietistischen Manövrierens zwischen hingenommenen Notwendigkeiten. Aufgegeben wird dabei der Versuch, eine Einheit zwischen unversöhnlichen Widersprüchen wie Erkennen und Handeln, Sein und Sollen, Beschreiben und Befehlen herstellen zu wollen. Indem die Notwendigkeiten hingenommen werden, werden sie zugleich ausgeklammert und der Einheitsversuch reduziert sich auf die Selbstkontrolle. ${ }^{12}$

12 Wieweit Wilhelm Schmid ein typischer Vertreter des Stoizismus in der Gegenwart ist, wäre eine Untersuchung wert. Da wir unter ähnlichen Bedingungen leben wie im Hellenismus, stoßen seine 
Auch der bereits erwähnte Ratschlag von Peirce, vom „lebendigen“ anstatt vom papierenen Zweifel auszugehen, trägt die quietistische Tendenz bereits in sich (auch wenn Peirce selbst sich nicht an sie gehalten hat). Der lebendige könnte der erwartbare, gewöhnliche, durchschnittliche Zweifel sein, dem dann ein papierener Zweifel durchaus gut täte. Der Pragmatismus als der allgemeinste Versuch, die Philosophie praktisch zu „rekonstruieren“ (Dewey), ist mit dem Problem konfrontiert, worin sein Programm denn noch bestehen soll, wenn die kritische Arbeit getan ist, den praktisch kontextlosen Gebrauch von Begriffen wie Geist, Wahrheit, Bedeutung, Moral offen zu legen. Vielleicht können wir auf einige dieser Begriffe, wie insbesondere Rorty meint, auch verzichten. Umso mehr stellt sich dann aber die Frage, was für die allgemeine Reflexion weiter zu tun bleibt. Rorty hat sich diese Frage selbst ebenfalls gestellt und auf die Bedeutung der Kunst verwiesen. Damit schließt er sich einem der drei anthropologischen Philosophen, an Nietzsche an, der die Kunst ebenfalls als die einzig verbleibende Möglichkeit sieht, eine Lebenseinheit herzustellen. Rorty beschränkt diesen Vorschlag realistischerweise auf das private Leben, denn wenn er außerdem die Literatur als Ersatz für die Ethik vorschlägt, so vorrangig wegen ihrer Erkenntnis- und nicht Selbstgestaltungsfähigkeit. Insgesamt ist Rortys Angebot quietistisch, weil er alle Erkenntnis auf soziale Bedingungen reduzieren will und das soziale Zusammenleben für problemlos hält, wenn es nur die intellektuell anspruchslose Forderung nach Freiheit beachtet. ${ }^{13}$

Wenn man sich fragt, ob Rorty so etwas wie ein Einheitsproblem sieht und löst, so ist die Antwort, dass die Einheit hergestellt werden soll von Seiten sozialer Übereinkünfte, weil alle Erkenntnisfragen letztlich praktisch-soziale Fragen sind. Im Unterschied zu Onora O'Neills, vielleicht Kants, aber sicher auch Habermas' normativer Einheitsidee soll sich das rein negativ, durch die „pragmatistische Aufklärung“ als der verbreiteten Einsicht in die völlig soziale Rolle der metaphysischen Ideen ergeben. Aus dieser Aufklärung entspringen dann Freiheit und friedliches Zusammenleben. Dieses Szenario leidet unter einem, für einen Pragmatisten erstaunlichen Ausmaß an Abstraktion, weil das der Philosophie zugeschriebene Bedürfnis, Metaphysik zu produzieren, einfach als auflösbar hingestellt wird. Ähnlich wie bei den Religionskritikern des 19. Jahrhunderts wird angenommen, dass die Genealogie das Bedürfnis problemlos und vollständig auflöst.

Wenn man sich jetzt fragt, wie die Einheit in der Philosophie auf eine nicht-quietistische, nicht letztlich ratlos werdende Weise beschaffen sein könnte, dann müssen wir zuerst noch einmal die zu beachtenden Punkte versammeln. Erstens, ein Bedürfnis nach und die Möglichkeit zu Einheit gibt es in der Philosophie nur von außerphilosophischen Motiven her. Zweitens, eine Kritik der Philosophie, soweit sie das verkennt, ist möglich, tendiert aber zum Quietismus. Drittens, einige anthropologische Ansätze liegen vor, in denen die außerphilosophischen Einheitsmotive systematisiert werden. Allerdings konkurrieren sie untereinander und tendieren teilweise selbst zu metaphysischen Annahmen. Viertens, von Seiten rein intellektueller Angebote ist keine Einheit zu erwarten, sie bergen immer die Gefahr der Zerstreuung.

Ohne Annahmen inhaltlicher Art, also mit einer bloßen Methode, bleibt diese Situation aussichtslos. Ich glaube aber, dass der Fundus des Pragmatismus durchaus eine Lösung parat hat, in diesem Fall eine abgeschwächte Version dessen, was man häufig „Deweys Metaphy-

Vorschläge auf das Interesse eines gegenüber der Fachphilosophie skeptisch gewordenen Publikums.

13 Ich beziehe mich insbesondere auf: R. Rorty, Contingency, Irony and Solidarity, Cambridge 1989; zum Quietismus vgl. ders., Naturalism and Quietism, in: ders., Philosophy as Cultural Politics. Philosophical Papers, Bd. 4, Cambridge 2007. 
sik“ nennt. ${ }^{14}$ Die zwei wichtigsten Punkte ergeben sich bereits aus dem, was bisher erwähnt wurde. Einerseits kann sich das Verständnis des Einheitsbedürfnisses nur auf die Lebensumstände im Allgemeinen beziehen, und das ohne eine bereits vorgefasste philosophische Interpretation. Daraus folgt die Bedeutung so genannter ,naturalistischer“, die ,natürlichen“ menschlichen Eigenschaften betreffenden Analysen. Auf der anderen Seite will ich hypothetisch daran festhalten, dass die Vereinbarkeit des individuellen mit dem sozialen Leben die Quelle der wichtigsten Konflikte ist, die sich für Menschen innerhalb wie außerhalb der Philosophie stellen. Dem entspricht auch bereits die pragmatistische Grundidee, die Philosophie an die reale Praxis zurückzubinden. Wenn das aber nicht im Quietismus enden soll, muss es konstruktiv praktiziert werden.

Anzuknüpfen ist dabei an die kritische Diagnose. Die Philosophie bleibt einzigartig geschickt darin, die Widersprüche in den entsprechenden Bereichen, wie etwa Determinismus und Freiheit, Körper und Geist, Vergangenheit und Gegenwart, früheres Ich und gegenwärtiges Ich, als Ausdruck eines biographischen Problems, oder privates und öffentliches Selbst, Autonomie und Zwang, Begründung und Wahrheit als Ausdruck eines sozialen Problems zu behandeln und diese Begriffspaare so umzuinterpretieren, dass sie als soziales Problem sichtbar und bearbeitbar werden. Beispiele für ein solches Uminterpretieren sind etwa Frankfurts Setzen der Sorge an die Stelle von Freiheit, oder Rawls' Erklären von Gerechtigkeit mittels Stabilität.

Beide Beispiele zeigen, dass die praktisch motivierte Kritik der Metaphysik nicht gelingen wird, solange sie nur eliminiert, also zentrale unserer philosophischen Begriffe ersatzlos aufgeben will. (Analoges dürfte auch für die Religion gelten, weil die Religion ebenso wie die Philosophie auf ein tiefes menschliches Bedürfnis nach Einheit antwortet, das nicht einfach aufgelöst werden kann.) Wie die Beispiele der Sorge und der Gerechtigkeit zeigen, geht es dabei nicht darum, grundsätzlich neue Ideen einzuführen, sondern natürliche Bedürfnisse angemessen zu beleuchten. Persönliche Freiheit wird nur über ein stimmiges Selbstbild schlüssig, das ein Gefühl erweitert. Gerechtigkeit ist nicht über Wahrheit, sondern über Konfliktminimierung explizierbar. Beides sind nicht-reduktiv naturalistische Vorschläge, weil sie den Ideen eine Eigenständigkeit einräumen, die sie aber praktisch unter Beweis stellen müssen.

Ähnlich wie bei Kants regulativen Ideen wird die Philosophie dann mit praktischen Zwecken verbunden, die ebenfalls unauflösbare Spannungen untereinander aufweisen, jetzt allerdings aus realen menschlichen Eigenschaften entspringen und nicht aus einer abstrakten Forderung nach Unendlichkeit. Die Zwecke des individuellen Lebens und des sozialen Zusammenlebens lassen sich nicht aufeinander reduzieren, und ihr Konfliktpotenzial vermehrt sich bei steigender Freiheit (also in der Zukunft). Der Zweck des individuellen Lebens ist eine Versöhnung mit sich, der des sozialen die gewinnbringende Kooperation. Das erste Ziel ist auch außerhalb, und notfalls gegen die Kooperation, verfolgbar. Deshalb ist die für die Philosophie letztlich einheitsstiftende Frage, wenn es eine einzige geben soll, die nach der Vereinbarkeit des individuellen Lebens mit dem sozial-kooperativen. Das ist sicher auch Kants zentrale ethische Frage, aber im Unterschied zu ihm ist es für den Nichtmetaphysiker mit dem Verweis auf die Vernunft nicht getan.

Lässt sich dieser Überblick in eine irgendwie verständliche Antwort auf die Motivfrage der Anfängerin in der Philosophie ummünzen, die ihr einen Weg von ihrem bereits laufenden Leben in die Philosophie bahnen könnte? Ein Versuch könnte so aussehen. Die Philosophie ist

14 J. Dewey, Experience and Nature, New York 1925; R. Rorty, Dewey’s Metaphysics, in: ders., Consequences of Pragmatism, Minneapolis 1982. 
das Experimentierfeld, auf dem wir bewusst oder unbewusst die existenziellen Probleme des persönlichen und sozialen Lebens in Gedankenkonflikte transformieren. Ohne ein gewisses Ausmaß dieser Transformation kommen wir nicht aus, wenn wir nicht ein völlig distanzloses und damit unfreies Leben führen wollen. Die spannende Aufgabe ist, das Maß an Philosophie herauszufinden, ohne das man den Konflikt zwischen sich und dem Allgemeinen nicht aushält, oder anders, in einem naheliegenden Verständnis, ohne das man nicht glücklich sein kann.

Prof. Dr. Anton Leist, Universität Zürich, Arbeits- und Forschungsstelle für Ethik, Zollikerstrasse 117, 8008 Zürich, Schweiz

\begin{abstract}
Today philosophy falls apart into a "theoretical" and a "practical" sub-discipline, a situation to the detriment of both. On the back of a sketch of three stages of Western philosophy a diagnosis is made as to the practical origin of the theoretical/practical split, which also already contains within it the clue for a therapy. Both parts of philosophy could be set on a convergent path by recognizing two practical conflicts underlying philosophical problems: the biographical conflict of accepting the world and the social conflict of accepting others. A non-reductivist study of these conflicts might mitigate the present alienation within philosophy.
\end{abstract}

